\title{
Influence of a non-ionic amphiphilic copolymer on the self-assembly of a peptide amphiphile that forms nanotapes
}

Article

Accepted Version

Dehsorkhi, A., Castelletto, V., Hamley, I. and Lindner, P. (2012) Influence of a non-ionic amphiphilic copolymer on the selfassembly of a peptide amphiphile that forms nanotapes. Soft Matter, 8 (33). pp. 8608-8615. ISSN 1744-683X doi: https://doi.org/10.1039/C2SM25990G Available at https://centaur.reading.ac.uk/28945/

It is advisable to refer to the publisher's version if you intend to cite from the work. See Guidance on citing.

To link to this article DOI: http://dx.doi.org/10.1039/C2SM25990G

Publisher: Royal Society of Chemistry

All outputs in CentAUR are protected by Intellectual Property Rights law, including copyright law. Copyright and IPR is retained by the creators or other copyright holders. Terms and conditions for use of this material are defined in the End User Agreement.

www.reading.ac.uk/centaur 
Central Archive at the University of Reading

Reading's research outputs online 
Influence of a Non-Ionic Amphiphilic Copolymer on the Self-Assembly of a Peptide Amphiphile that Forms Nanotapes

\author{
A. Dehsorkhi, V. Castelletto, I. W. Hamley* \\ School of Chemistry, Pharmacy and Food Biosciences, University of Reading, Reading RG6 \\ 6AD, U.K. \\ P. Lindner \\ Institut Laue-Langevin, BP 156, 6, rue Jules Horowitz, 38042 Grenoble Cedex 9, \\ France
}

For Soft Matter. Revised 29/6/12.

* Author for correspondence. Email: I.W.Hamley@ reading.ac.uk; tel: + 441183788453 


\begin{abstract}
The influence of a non-ionic polymeric surfactant on the self-assembly of a peptide amphiphile (PA) that forms nanotapes is investigated using a combination of microscopic, scattering and spectroscopic techniques. Mixtures of Pluronic copolymer P123 with the PA $\mathrm{C}_{16}$-KTTKS in aqueous solution were studied at a fixed concentration of the PA at which it is known to self-assemble into extended nanotapes, but varying P123 concentration above and below the critical micelle concentration. We find that P123 can disrupt the formation of $\mathrm{C}_{16^{-}}$ KTTKS nanotapes, leading instead to cylindrical nanofibril structures. The spherical micelles formed by P123 at room temperature are disrupted in the presence of the PA. There is a loss of cloudiness in the solutions as the large nanotape aggregates formed by $\mathrm{C}_{16}$-KTTKS are broken up, by P123 solubilization. At least locally, $\beta$-sheet structure is retained, as confirmed by XRD and FTIR spectroscopy, even for solutions containing $20 \mathrm{wt} \% \mathrm{P} 123$. This indicates, unexpectedly, that peptide secondary structure can be retained in solutions with high concentration of non-ionic surfactant. Self-assembly in this system exhibits slow kinetics towards equilibrium, the initial self-assembly being dependent on the order of mixing. Heating above the lipid chain melting temperature assists in disrupting trapped nonequilibrium states.
\end{abstract}




\section{Introduction}

Peptide amphiphiles (PAs) or lipopeptides are currently attracting considerable interest due to their ability to assemble into high aspect ratio nanostructures under certain conditions such as

$\mathrm{pH}$, temperature and ionic strength. ${ }^{1-4} \mathrm{PA}$ molecules normally consist of two main regions. ${ }^{1-7}$ The first of these regions is a hydrophobic alkyl tail or lipid chain, which is attached to a hydrophilic peptide sequence. In aqueous solution, the hydrophobic tail will aggregate for self-assembly to take place, while the peptide region presents its functional group on the surface of the nanostructure to confer remarkable properties of bioactivity. ${ }^{1-4}$ The peptide epitope can play an important role in biological processes such as cell signal transduction, cell adhesion in the extra cellular matrix (ECM), cell growth and cell mobility.

Amphiphilicity promotes self-assembly due to the driving force arising from interactions involving polar and apolar regions of the molecules. PA molecules tend to aggregate in order to avoid interactions with the surrounding aqueous environment. This occurs via exposure of hydrophilic regions in contrast to the hydrophobic domain which is buried within the nanostructure. These PA molecules can self-assemble in aqueous solution to form different nanostructures such as peptide bilayers, nanofibres, nanotapes, ribbons and micelles. ${ }^{3-7}$

In solution, PA aggregation is also influenced by intermolecular hydrogen bonding which leads to the formation of $\beta$-sheets. Previous literature has shown that the peptide domain of PA can be designed to consist of alternating hydrophilic and hydrophobic amino acids to favour $\beta$-sheet formation, along with additional spacer or biofunctional motifs. Peptide 
amphiphiles can assemble into extended fibrillar structures due to the existence of intermolecular hydrogen bonds. ${ }^{4,5,9-11}$ In one case, PA $\mathrm{C}_{16} \mathrm{O}-\mathrm{VEVE}$ was shown to selfassemble into tapes, also termed "nanobelts". ${ }^{10}$ By using a peptide sequence of four amino acids with alternating polarity it was possible for the PA molecule to self-assemble into long flat nanobelt structures. Adams et al have reported the self-assembly of short surfactant-like peptides (SLP) into broad tape-like fibers. ${ }^{8}$ The SLP consisted of six hydrophobic valine residues making up the hydrophobic tail, which was attached to a head group containing two negatively charged aspartic acid residues. It was revealed by FTIR spectroscopy that $\beta$-sheets were present, which gives rise to the characteristic tape-like structures. ${ }^{8}$ Aulisa et al. have investigated the self-assembly of a short designer PA into micelles, this system having the ability to inhibit proliferation of pancreatic cancer cells. ${ }^{12}$ This PA was mixed with an anionic surfactant, sodium dodecyl sulphate (SDS). When mixed with SDS, it was revealed by circular dichroism that there was a change from a weak $\alpha$-helix to a moderate $\alpha$-helix content.

Recently our group investigated the self-assembly of a PA, which consists of a pentapeptide attached to a hexadecyl lipid chain. It was shown that the collagen stimulating $\mathrm{PA} \mathrm{C}_{16^{-}}$ KTTKS (which is incorporated in a range of commercial products used in skincare applications with tradename Matrixyl ${ }^{\mathrm{TM}}$ ) forms extended nanotapes with a bilayer structure. ${ }^{13}$ In a related study, the influence of the anionic surfactant SDS on the self-assembly of this PA was examined. ${ }^{14}$ A strong influence of electrostatic interactions was noted, leading to several nanostructures depending on the amount of added SDS, this in turn led to distinct macroscopic properties such as gel formation. Here, we investigate the binding of a non-ionic surfactant to a PA $\mathrm{C}_{16}$-KTTKS and its effects on self-assembly. Our aim is to examine the role of co-operativity in self-assembly and to examine the influence of uncharged surfactant on the aggregation of a PA containing a cationic peptide headgroup. We chose to use one of 
the widely used and studied Pluronic copolymers, P123. Pluronic copolymers are amphiphilic poly(ethylene oxide)-poly(propylene oxide)-poly(ethylene oxide) [PEO-PPO-PEO] triblock copolymers, and these are readily available commercially, have already been studied extensively and have useful properties including biocompatibity. ${ }^{16,17}$

The PA $\mathrm{C}_{16}$-KTTKS consists of a 16 carbon (palmitoyl) chain $\left(\mathrm{C}_{16}\right)$ attached to a pentapeptide sequence, KTTKS, which is derived from Type I procollagen. This lipopeptide is used in cosmetic applications such as anti wrinkle creams. ${ }^{13,18}$ It is claimed that the pentapeptide sequence, KTTKS boosts the production of the extracellular matrix in fibroblasts via collagen and fibronectin production. ${ }^{18}$ In formulations, $\mathrm{C}_{16}$-KTTKS is mixed with surfactants and it is therefore of practical, as well as fundamental interest to examine self-assembly in mixtures with surfactants such as the non-ionic copolymers studied here.

\section{Experimental Methods}

\section{Materials and Sample Preparation}

Peptide amphiphile $\mathrm{C}_{16}$-KTTKS, Palmitoyl-Lys-Thr-Thr-Lys-Ser was purchased from CS Bio (Menlo Park, California) and was in the form of the acetate salt. Two different batches were used. For the first, the purity was $97.6 \%$ by analytical HPLC, MS 802.47 (expected) 802.05 (measured), acetate content was $11 \%$ (by HPLC). For the second batch, the purity was $98.61 \%$ by analytical HPLC, MS 802.5 (expected) 802.2 (measured), acetate content was $11.61 \%$ (by HPLC). 
Pluronic P123 was obtained as a gift from the manufacturer (BASF). It has the formula $\mathrm{EO}_{20} \mathrm{PO}_{72} \mathrm{EO}_{20}$ where the uncertainty in the number of ethylene oxide (EO) and propylene oxide (PO) units is $\pm 2 .{ }^{17}$ The molar mass is quoted in the range $5750-5800 \mathrm{~g} \mathrm{~mol}^{-1}$.

The PA was dissolved in water. The measured $\mathrm{pH}$ of a $1 \mathrm{wt} \%$ solution (the concentration for the majority of the measurements) was $\mathrm{pH} 4.5$.

For the initial studies, $\mathrm{C}_{16}$-KTTKS was weighed out followed by P123. Water was then added to make up a solution with a defined concentration. A vial containing the solution was then placed in a sonicator at $30{ }^{\circ} \mathrm{C}$ for approximately one hour, in order to dissolve both $\mathrm{C}_{16^{-}}$ KTTKS and P123 in water.

For studies on the equilibration of samples at room temperature, two protocols were followed. In the first, a $1 \mathrm{wt} \%$ solution of $\mathrm{C}_{16}$-KTTKS was prepared and left to stand for a week (in the fridge) to allow for sufficient time for self-assembly to occur. This was then followed by the addition of P123 to make up a $1 \mathrm{wt} \% \mathrm{C}_{16}$-KTTKS solution with either $5 \mathrm{wt} \%$ or $20 \mathrm{wt} \%$ of P123. SAXS was performed immediately after preparing the mixture and again two months later to study the effects of ageing. In the second procedure, solutions of P123 was prepared first and left for a week, followed by the addition of $1 \mathrm{wt} \% \mathrm{C}_{16}$-KTTKS.

To allow for thermal equilibration above the lipid chain melting temperature (previously reported to occur at around $40-45{ }^{\circ} \mathrm{C}^{13,14}$ ), SAXS measurements were also performed for samples prepared according to the different mixing protocols, heated to $60{ }^{\circ} \mathrm{C}$ and cooled back to room temperature. 
Spectra were acquired on a Bruker AVANCE $\mathrm{II}^{+}$Cryo Probe $500 \mathrm{MHz}$ spectrometer. All spectra were recorded at a temperature of $25{ }^{\circ} \mathrm{C}$. All ${ }^{1} \mathrm{H}$ NMR spectra were obtained for 1 wt $\% \mathrm{C}_{16}$-KTTKS on its own and mixed with $5 \mathrm{wt} \%$ and $20 \mathrm{wt} \% \mathrm{P} 123$ in $\mathrm{D}_{2} \mathrm{O}$. The solutions were placed in a NMR tube with a cap on top to prevent leaking/evaporation of the samples during the measurement.

Circular Dichroism (CD) and Linear Dichroism (LD) Spectroscopy

The CD and LD spectra were recorded using a Jasco (Great Dunmow, Essex) J-815 spectropolarimeter. Solutions of the PA (1 wt $\%)$ with varying concentrations of P123 were loaded in parallel plaque cells (Hellma quartz Suprasil@), with a $0.01 \mathrm{~mm}$ pathlength. The $\mathrm{CD}$ and $\mathrm{LD}$ data were measured using $1 \mathrm{sec}$ acquisition time per point and $0.5 \mathrm{~nm}$ step. The post-acquisition smoothing tool from Jasco Spectra Manager software was used to remove random noise elements from the averaged spectra. A residual plot was generated for each curve in order to verify whether or not the spectrum has been distorted during the smoothing process. The CD and LD signal from the water background was subtracted from the CD and LD data of the peptide solutions.

\section{Fourier Transform Infra-Red (FTIR) Spectroscopy}

Spectra were recorded using a Nexus-FTIR spectrometer equipped with a DTGS detector and a multiple reflection attenuated total reflectance (ATR) system. Solutions of $1 \mathrm{wt} \% \mathrm{C}_{16^{-}}$ KTTKS with varying concentrations of $\mathrm{P} 123$ in $\mathrm{D}_{2} \mathrm{O}$ were sandwiched in ring spacers between two $\mathrm{CaF}_{2}$ plate windows (spacer $25 \mu \mathrm{m}$ ). All spectra were scanned 128 times over the range $4000-950 \mathrm{~cm}^{-1}$. 
X-ray diffraction was performed on a stalk prepared from $1 \mathrm{wt} \%$ solutions of C16-KTTKS with varying amounts of P123. The stalk was mounted (vertically) onto the four axis goniometer of a RAXIS IV++ X-ray diffractometer (Rigaku) equipped with a rotating anode generator. The XRD data was collected using a Saturn 992 CCD camera.

\section{Transmission Electron Microscopy (TEM)}

To prepare specimens for TEM, a droplet of PA + P123 solution ( 1 wt $\% \mathrm{C}_{16}$-KTTKS +0.2 wt $\%$ or $20 \mathrm{wt} \% \mathrm{P} 123$ ) was placed on a $\mathrm{Cu}$ grid coated with a carbon film (Agar Scientific, UK), stained with uranyl acetate (1 wt \%) (Agar Scientific, UK) and dried. TEM experiments were performed using a JEOL JEM-2010 microscope operated at $200 \mathrm{kV}$. Images were recorded using a Gatan Orius digital camera. The material was found to be beam sensitive, so only a low-intensity beam was used.

\section{Small-Angle X-ray Scattering (SAXS)}

SAXS was performed using a Bruker Nanostar instrument using $\mathrm{CuK}_{\alpha}$ radiation from an Incoatec microfocus source. The beam was collimated by a three slit system. The sample was mounted in a glass capillary ( $1 \mathrm{~mm}$ diameter). The sample-detector distance was $105 \mathrm{~cm}$ and a Vantec-2000 photon counting detector was used to collect SAXS patterns. The wavenumber $q=4 \pi \sin \theta / \lambda$ (where $2 \theta$ is the scattering angle and $\lambda=1.54 \AA$ is the wavelength) scale was calibrated using silver behenate. For the thermal equilibration experiments, a water-bath heating unit was connected to the sample holder. The samples were heated from room temperature to $60{ }^{\circ} \mathrm{C}$ at a ramp rate of $10{ }^{\circ} \mathrm{C}$ per minute and held at that temperature for 30 minutes prior to SAXS. This was then followed by cooling the samples back to room temperature at which point another SAXS measurement was obtained. 
SANS was performed on instrument D11 at the Institut Laue-Langevin, Grenoble, France. The sample-to-detector distance was $8 \mathrm{~m}$, with an $8 \mathrm{~m}$ collimation length and a wavelength $\lambda$ $=4.51 \AA$ A. Samples containing mixtures of $1 \mathrm{wt} \% \mathrm{C}_{16}$-KTTKS with $\mathrm{P} 123$ in $\mathrm{D}_{2} \mathrm{O}$ were placed in a quartz couette cell comprising concentric cylinders with a rotating outer cylinder and a fixed inner bob. The sample gap was $0.5 \mathrm{~mm}$. The sample was subjected to steady shear, at varying shear rates.

\section{Results and Discussion}

\section{Microscopic and Scattering Studies on Self-Assembled Structures}

We study the addition of the Pluronic P123 to $\mathrm{C}_{16}$-KTTKS and investigate whether a nonionic surfactant has any effect on the self-assembled structure of the PA. A concentration of $1 \mathrm{wt} \% \mathrm{C}_{16}$-KTTKS in a solution of water was selected for this investigation as it is known from previous work that the PA forms extended fibrillar structures (predominantly nanotapes) under these conditions. ${ }^{12}$ P123 concentrations were selected, spanning the critical micelle concentration $c m c=0.09-0.18 \mathrm{wt} \%$ at $20{ }^{\circ} \mathrm{C} \cdot{ }^{17,19,20} \mathrm{P} 123$ shows a transition to a micellar gel structure at room temperature at around $25 \mathrm{wt} \%$ in water, ${ }^{21}$ however the $\mathrm{P} 123$ concentration was kept below this value. The copolymer is expected to form spherical micelles at room temperature, however as with other Pluronics, the formation of cylindrical micelles is favoured at high temperature. ${ }^{16,17}$

Fig.1 illustrates the appearance of the samples, a $1 \mathrm{wt} \%$ solution of $\mathrm{C}_{16}-\mathrm{KTTKS}$ or solutions with lower concentrations of added P123 have a milky appearance. This results from light

scattering from large aggregates. As shown previously, ${ }^{13} \mathrm{C}_{16}$-KTTKS forms highly extended 
nanotapes (persistence length $>1 \mu \mathrm{m}$ ) and this causes light scattering. On addition of P123, the sample becomes translucent (10 wt $\%$ added P123) or transparent (20 wt $\%$ added P123). This points to a solubilizing effect of the added non-ionic polymeric surfactant, which appears to break up larger clusters of nanotapes. This was investigated further by TEM and SAXS, as described shortly. A key question is whether this is accompanied by loss of peptide secondary structure, and this was examined by CD and FTIR spectroscopy. The ability to reduce clouding (which is accompanied by slow precipitation of some of the $\mathrm{C}_{16}$-KTTKS aggregates) in the solution (whilst retaining peptide secondary structure) may be useful in applications requiring a long shelf-life or a transparent appearance.

TEM images from solutions of $\mathrm{C}_{16}$-KTTKS with either low $(0.2 \mathrm{wt} \%)$ or high $(20 \mathrm{wt} \%)$ concentrations of P123 are shown in Fig.2 (a further image for a sample containing $25 \mathrm{wt} \%$ P123 is shown in SI Fig.1). The image for $1 \mathrm{wt} \% \mathrm{C}_{16}$-KTTKS mixed with $0.2 \mathrm{wt} \% \mathrm{P} 123$ reveals tape structures with an extended persistence length, which is essentially consistent with our previous results for $\mathrm{C}_{16}$-KTTKS itself. ${ }^{13,14}$ The addition of further P123 (e.g. 20 wt\% P123, Fig. 2b) leads to change of morphology, from nanotapes to predominantly cylindrical fibres with lengths ranging from 5-10 $\mu \mathrm{m}$ or more. As P123 concentration increases the fibres tend to become longer and thinner however a few nanotape structures are still present as shown in Fig. 2b. The reduction in the proportion of nanotapes with increasing P123 concentration appears to correlate to the reduction in cloudiness of solutions illustrated in Fig.1.

The nanostructures of the $\mathrm{C}_{16}$-KTTKS/P123 mixtures were further investigated by employing both small angle neutron scattering (SANS) and small angle X-ray scattering (SAXS). SANS was used to obtain additional information regarding the self-assembled nanostructure. Fig.3 
shows intensity profiles obtained for a solution containing $1 \mathrm{wt} \% \mathrm{C}_{16}$-KTTKS and $20 \mathrm{wt} \%$ P123. Data are presented for samples under shear, and also at rest. Shear was applied in order to examine possible flow-induced alignment of nanotape or nanofibril structures. In fact, there were no differences in the SANS data in the absence or presence of shear (up to a shear rate $=125 \mathrm{rpm}$ ) as shown by the profile in Fig.3 measured for a sample with high P123 content, and lack of orientation in 2D patterns (results not shown). Potential alignment was also examined by linear dichroism, as discussed shortly. The oscillations in the SANS intensity profile produced by the form factor for the $1 \mathrm{wt} \% \mathrm{C}_{16}-\mathrm{KTTKS}+20 \mathrm{wt} \% \mathrm{P} 123$ were fitted to a cylindrical form factor model with length $L$, radius $R_{0}$ and scattering contrast $\Delta \eta$. The following expression, allowing for Gaussian polydispersity $\sigma$ in radius, was used to fit the data ${ }^{26}$

$I(q)=k \int_{0}^{\infty} \int_{0}^{1} \exp ^{-\left(\frac{R-R_{0}}{2 \sigma^{2}}\right)^{2}} \times\left(\frac{J_{1}\left(q R \sqrt{\left.1-x^{2}\right)} \sin (q L x / 2)\right.}{q^{2} R \sqrt{1-x^{2}} L x}\right) d x d R$.

Here $k$ is a constant defined as:

$k=16\left(\pi R_{0}^{2} L\right)^{2} \Delta \eta^{2} \sqrt{\frac{\pi}{2}} \sigma\left(1+\operatorname{erf}\left(\frac{R_{0}}{\sqrt{2 \sigma}}\right)\right)$.

Due to the extended nature of the fibrils (as observed by TEM) the length was fixed at $L=$ $100 \mathrm{~nm}$ while the radius was varied with a fitted value $R=4.9 \mathrm{~nm}$. Since $L \gg R$ the length does not influence the shape of the form factor, the actual persistence length being larger as evident from Fig.2, however a lower value is numerically convenient. A constant background 
term was applied in order to fit the data, with a value $\mathrm{BG}=0.214$. The polydispersity in the radius of the cylindrical fibres was found to be $16 \%$.

SAXS data are presented in Fig.4. These show the essential feature already reported ${ }^{13,14}$ for $\mathrm{C}_{16}$-KTTKS in aqueous solution, i.e. a Bragg peak at $q=0.122 \AA^{-1}$, corresponding to a spacing $d=52 \AA$. This is the spacing of bilayers of the PA. On addition of 5 wt $\%$ P123 or more, form factor oscillations progressively appear. As noted above, and quantitatively analysed in the SANS data, these correspond to the formation of cylindrical fibrillar structures. The $\mathrm{C}_{16}$-KTTKS bilayer peak reduces in intensity as P123 concentration increases, and merges with form factor oscillations for $10 \mathrm{wt} \%$ and $20 \mathrm{wt} \%$ P123 samples. For comparison, a SAXS profile for a $10 \mathrm{wt} \%$ P123 solution is included in Fig.4, this confirms the absence of the bilayer spacing peak, and the presence of the form factor oscillations, as well as structure factor features which occur at this high concentration, especially at low $q$. The form factor of P123 spherical micelles has been investigated in detail previously. ${ }^{15}$ Our results are consistent with TEM, i.e. the SAXS and SANS data show features of a fibril form factor at high P123 concentration, and the loss of features associated with the bilayer ordering of the P123 in the nanotapes. The spherical micelles formed by neat P123 are disrupted in the presence of $\mathrm{C}_{16}$-KTTKS and instead cylindrical micelles are formed.

X-ray diffraction on stalks prepared by drying solutions was used to investigate the presence of $\beta$-sheet structures. Fig.5 shows a selected fibre XRD pattern. Table 1 summarizes the observed reflections. The main trend that can be noted is the progressive disappearance of the large $d$ spacing equatorial reflections as P123 concentration increases, while the strong meridional $4.75 \AA$ reflection arising from the $\beta$-strand spacing is retained. ${ }^{1}$ The equatorial long spacings arise from the bilayer spacing and the stacking of $\beta$-sheets which is disrupted 
as P123 concentration is increased. This occurs concomitantly with the transition from tapes to fibrils observed by TEM, SANS and SAXS. However, even at the highest P123 concentration, the $\beta$-sheet structure is retained locally. PEO crystallization on drying the P123-containing solution ${ }^{23,24}$ was not observed, presumably due to the low molar mass of the PEO.

Spectroscopic Techniques to Peptide Secondary Structure

Having established the presence of fibrillar structures by TEM, SANS, SAXS and XRD, spectroscopic methods were also used to investigate the presence of $\beta$-sheet features. FTIR confirms that $\beta$-sheet structures are present within the mixtures with a peak at $1609 \mathrm{~cm}^{-1}$ within the amide I' band as shown in Fig. 6. This is consistent with our previous reports. ${ }^{13,14}$ The peak is retained on increasing P123 concentration over the range examined, however for $10 \mathrm{wt} \%$ P123 a peak near $1650 \mathrm{~cm}^{-1}$ associated with disordered structure develops, pointing to the loss of some $\beta$-sheet ordering. This peak position is considered to be slightly out of the usual range for $\beta$-sheet structures and this could be caused by the attachment of the hexadecyl lipid chain, which is attached to the pentapeptide head group. No peaks were observed in the antiparallel $\beta$-sheet frequency of $1675-1695 \mathrm{~cm}^{-1}$ therefore it can be assumed that these $\beta$ sheets have a parallel arrangement. ${ }^{25}$

$\mathrm{CD}$ data as shown in Fig. 7a reveal a minimum at $222 \mathrm{~nm}$ for $1 \mathrm{wt} \% \mathrm{C}_{16}-\mathrm{KTTKS}$, which is characteristic of a $\beta$-sheet structure as confirmed by FTIR results. This result is very similar to our previous findings i.e $\beta$-sheet signature at $217 \mathrm{~nm} .{ }^{13}$ The CD spectra show a gradual transition from $\beta$-sheets to random coils with increasing concentration of P123 as the minimum at $222 \mathrm{~nm}$ shifts to $199 \mathrm{~nm}$. This indicates disruption to the $\beta$-sheet superstructure 
upon addition of P123, however there is still a minimum or shoulder around $220 \mathrm{~nm}$. indicating the presence of $\beta$-sheets. XRD and FTIR data also show that $\beta$-sheet structures are still present locally even for $20 \mathrm{wt} \% \mathrm{P} 123$.

To examine possible flow alignment contributions to the CD spectra, linear dichroism spectra were measured and the results are shown in Fig.7b. The LD spectra were measured for samples loaded into parallel plaque cells, and therefore any alignment results from shear during loading. LD reveals alignment of a solution of $1 \mathrm{wt} \% \mathrm{C}_{16}$-KTTKS itself and also the solution containing $0.2 \mathrm{wt} \% \mathrm{P} 123$, however as the concentration of P123 increases a reduction in LD signal is observed. The alignment observed for solutions with low P123 concentration is consistent with shear alignment of nanotapes, as observed previously by SAXS. ${ }^{13,14}$ However, the samples containing $10 \mathrm{wt} \%$ P123 or $20 \mathrm{wt} \%$ P123 which contain fibrillar structures, do not exhibit flow alignment (as also indicated by SANS). As mentioned above, this suggests that the fibrils resemble unaligned, unentangled worm-like micelles. The sign of the linear dichroism, $\mathrm{LD}=A_{\|}-A_{\perp}$, in the spectra (where $A_{\|}$and $A_{\perp}$ are the absorbances parallel and perpendicular to the flow direction, respectively), provides information on the alignment of the backbone and aromatic units in $\mathrm{C}_{16}$-KTTKS. The $\pi$ - $\pi^{*}$ transition arising from the carbonyl bonds in the peptide backbone gives rise to the minimum at $195 \mathrm{~nm}^{26-28}$ The strong minimum observed for $\mathrm{C}_{16}$-KTTKS with no added P123 is ascribed to the peptide $\mathrm{n}-\pi^{*}$ transition at about $220 \mathrm{~nm}$. The fact that the LD signals are negative for the $\mathrm{C}_{16}$-KTTKS solution and the $\mathrm{C}_{16}$-KTTKS solution with low P123 concentration indicates alignment of $\mathrm{C}=\mathrm{O}$ bonds perpendicular to the alignment direction (along which the nanotapes are presumed to orientate). This is consistent with the arrangement of the peptide headgroups within the nanotapes, as discussed previously. ${ }^{13}$ 
To probe the binding of the PA with P123 at the molecular level, NMR spectroscopy measurements were performed. ${ }^{1} \mathrm{H}$ NMR spectra are shown in SI Fig. 2. Spectra were measured for $0.05 \mathrm{wt} \% \mathrm{P} 123,5 \mathrm{wt} \% \mathrm{P} 123,1 \mathrm{wt} \% \mathrm{C}_{16}$-KTTKS and mixtures of the two components to observe the interaction between P123 and $\mathrm{C}_{16}$-KTTKS. The spectrum for 5 wt\% P123 on its own exhibits a peak at $3.75 \mathrm{ppm}$, which is assigned to the $\mathrm{CH}$ units in PPO however this peak is absent in the case of P123 with a concentration of $0.05 \mathrm{wt} \%$. This is due to the fact that this concentration is below the critical micellar concentration of P123 (0.09 $0.18 \mathrm{wt} \%$, at $\left.20{ }^{\circ} \mathrm{C}\right) .{ }^{17,19,20}$ The P123 NMR spectrum obtained is consistent with previous studies. ${ }^{29}$ SI Fig.2b shows a ${ }^{1} \mathrm{H}$ NMR spectrum obtained from a $1 \mathrm{wt} \%$ solution of $\mathrm{C}_{16^{-}}$ KTTKS, along with assignments.

The NMR spectra of $\mathrm{C}_{16}$-KTTKS/P123 mixtures (SI Fig.2c) exhibit peaks from $\mathrm{C}_{16}$-KTTKS, which unfortunately generally overlap with peaks from P123. The main observation is in the region of peaks associated with the amide groups of $\mathrm{C}_{16}$-KTTKS $(6.5-8 \mathrm{ppm})$, which are modified upon the addition of P123 as shown in SI Fig. 2d. This is probably due to the enhanced H/D exchange of amide protons for some of the $\mathrm{NH}$ amides in the presence of P123. This is consistent with the formation of solvent accessible structures when the $\mathrm{C}_{16^{-}}$ KTTKS bilayers are disrupted by addition of P123.

\section{Mechanisms of Self-Assembly - Influence of Order of Mixing and Aging}

Additional experiments were carried out to further enhance our understanding of the mechanism of self assembly in mixtures of $\mathrm{C}_{16}$-KTTKS with P123. The effects of order of mixing were investigated for samples in which the components were mixed simultaneously before addition of water, compared to samples in which one of the components was added to 
an aqueous solution after the other. All of these studies were performed at room temperature. Aging studies were also performed, to elucidate possible slow kinetic processes. This included preparing a $1 \mathrm{wt} \%$ solution of $\mathrm{C}_{16}$-KTTKS and leaving it to stand for a week to allow sufficient time for self-assembly to occur, which was then followed by the addition of P123. SAXS was performed immediately after preparing the mixture and again two months later to study the effects of ageing. The same procedure was applied to a solution of P123 followed by the addition of $\mathrm{C}_{16}$-KTTKS.

Fig. 8a presents the SAXS profiles from the order of mixing and ageing studies on the $1 \mathrm{wt} \%$ $\mathrm{C}_{16}$-KTTKS + $5 \mathrm{wt} \%$ P123 sample. The SAXS curve for the sample for which the components were "mixed together" (simultaneously) before addition of water reveals the presence of the $\mathrm{C}_{16}$-KTTKS bilayer peak $\left(q^{*}=0.122 \AA^{-1}\right)$, which is also present for some of the other samples along with the observation of form factor oscillations. However the bilayer peak is attenuated when $\mathrm{C}_{16}$-KTTKS is added to a (micellar) solution of P123. This shows that the non-ionic surfactant solution can hinder the formation of PA fibrillar aggregates.

The SAXS profiles for the sample containing $1 \mathrm{wt} \% \mathrm{C}_{16}$-KTTKS $+20 \mathrm{wt} \% \mathrm{P} 123$ (Fig.8b) also exhibit the typical $\mathrm{C}_{16}$-KTTKS bilayer peak with form factor oscillations due to the formation of cylindrical fibrils upon addition of P123. The SAXS profile varies depending on the order of mixing and ageing protocol. A reduction or even elimination in some cases of the $\mathrm{C}_{16}$-KTTKS bilayer peak is noticed. This is ascribed to the ability of P123 micelles to solubilise $\mathrm{C}_{16}$-KTTKS and hinder formation of bilayers. There are also some shifts in the location of the cylinder form factor oscillations. For ease of comparison, all the SAXS profiles from the different mixing protocols for solutions with either $5 \mathrm{wt} \%$ added P123 or 20 wt\% added P123 are included in SI Fig.3. 
The SAXS data at low $q$ for representative data sets for solutions containing $5 \mathrm{wt} \%$ and 20 wt $\%$ P123 can be fitted to a cylinder form factor with an average radius $R=7 \mathrm{~nm}$, as shown by representative profiles in Fig.8c. The cylinder radius is larger than the value from the fitting to the SANS data shown in Fig. 3. Adding the PA to a (micellar) P123 solution causes the maximum in the SAXS profiles to shift to lower $q$ compared to the mixing together curve (Fig.8b) indicating a larger fibril radius. Comparison of cylinder dimensions from SAXS and SANS form factors will also be affected by the distinct contrasts for the two types of scattering experiment. The fit parameters using Equations 1,2 for $1 \mathrm{wt} \% \mathrm{C}_{16}$-KTTKS +5 wt $\%$ P123 with $\mathrm{C}_{16}$-KTTKS added later were as follows: $L=100 \mathrm{~nm}$ (fixed), $R=6.7 \mathrm{~nm}$ and $\Delta \eta=1.28 \times 10^{-6}$. A linear background was added, represented by an equation $1.79-10.4^{*} q$. The fitting parameters for the aged $1 \mathrm{wt} \% \mathrm{C}_{16}$-KTTKS with $20 \mathrm{wt} \% \mathrm{P} 123$ added later were $L$ $=500 \mathrm{~nm}$ (fixed), $R=7.4 \mathrm{~nm}, \Delta \eta=1.32 \times 10^{-6}$ and constant $\mathrm{BG}=0.6$.

Since aging does not lead to the same steady state self-assembled structure (compare the profiles for the aged samples in Fig.8a and Fig.8b), thermal annealing was examined. A temperature of $60{ }^{\circ} \mathrm{C}$ was selected, above the lipid chain melting transition. Fig.8d presents the measured SAXS profiles for samples prepared according to the three mixing protocols, both at $60{ }^{\circ} \mathrm{C}$ and after cooling to room temperature. The three profiles for the samples at 60 ${ }^{\circ} \mathrm{C}$ are very similar, as are the corresponding profiles for the cooled samples. This indicates that this protocol has successfully led to a reproducible final "equilibrium" state. This is believed to result from mobility, especially of the tails of the PA molecules, above the lipid chain melting temperatures. At both temperatures, form factor oscillations from cylinders are observed with no PA bilayer peaks. The cylindrical fibrils have a larger radius at $60{ }^{\circ} \mathrm{C}$ than at room temperature. Similar conclusions concerning equilibration, and fibril radius, were 
reached from SAXS profiles for $1 \mathrm{wt} \% \mathrm{C}_{16}$-KTTKS + $5 \mathrm{wt} \% \mathrm{P} 123$, the corresponding data being shown in SI Fig.4. Some variability in bilayer peak intensity was still noted, which is due to residual inhomogenenity in this sample (Fig.1b).

\section{Summary and Discussion}

We have shown that addition of the nonionic polymeric surfactant P123 can be used to prepare homogeneous transparent solutions of the bioactive peptide amphiphile $\mathrm{C}_{16}$-KTTKS. The $\beta$-sheet structure of $\mathrm{C}_{16}$-KTTKS is retained even with $20 \mathrm{wt} \%$ added P123, as shown by FTIR spectroscopy and X-ray diffraction. However, the nanotapes comprising peptide bilayers observed for $\mathrm{C}_{16}$-KTTKS itself in aqueous solution are progressively replaced by cylindrical fibrils upon addition of more P123, and there is a macroscopic loss of chirality due to progressive increase in PA random coil conformation, as shown by CD spectroscopy. The peptide amphiphile nanotapes undergo alignment under shear flow, as revealed by prior SAXS studies, ${ }^{13}$ and linear dichroism reported above. However, the cylindrical fibres observed for 5-20 wt\% do not exhibit shear alignment, as confirmed by SANS and LD, suggesting that these fibrils have a lower persistence length similar to non-entangled wormlike micelles. ${ }^{30,31}$ However, analysis of NMR peaks associated with the amide groups showed formation of solvent accessible structures when the $\mathrm{C}_{16}$-KTTKS bilayers are disrupted by addition of P123. Two possible mechanisms for the transition from nanotapes to fibrils on addition of the Pluronic copolymer come to mind. In the first, $\mathrm{C}_{16}$-KTTKS is solubilised within P123 cylindrical micelles, although with some degree of $\beta$-sheet order. In the second, cylindrical micelles (possibly containing some solubilized PA) coexist with $\mathrm{C}_{16^{-}}$ KTTKS nanotapes. The second alternative is actually favoured, firstly because TEM suggests coexisting structures (Fig.2) although predominantly fibrils as P123 concentration increases but secondly because geometric constraints make it unlikely that extended $\mathrm{C}_{16}$-KTTKS $\beta$ - 
sheet structures can exist within P123 cylindrical micelles. Considering that the concentration of P123 is much higher than that of $\mathrm{C}_{16}$-KTTKS and also taking account of the measured fibril radius and the molar mass of the core-forming PPO block of the copolymer, a convincing model for $\beta$-sheet assembly of the PA within P123 cylindrical micelles does not seem possible.

We have also shown that sample preparation conditions require careful design in order to achieve reproducible end states when preparing mixtures containing $\mathrm{C}_{16}$-KTTKS which forms inhomogeneous cloudy suspensions in $1 \mathrm{wt} \%$ aqueous solutions, due to the formation of clusters of the highly extended nanotapes. Pre-existing P123 micellar solutions reduce the capability of the peptide amphiphile to aggregate into bilayers. Thermal processing above the lipid chain melting transition temperature is required to generate reproducible equilibrium states. The enhanced mobility of the lipid chains presumably facilitates dynamic selfassembly, avoiding trapped non-equilibrium states. Aging appears to be insufficient to achieve this due to the locked-in ordered state of the lipid tail groups.

Our results provide insight into the self-assembly of PA/neutral surfactant mixtures potentially relevant to formulations of $\mathrm{C}_{16}$-KTTKS in existing cosmeceutical uses (we are currently examining the collagen production properties of this compound). Our findings are also valuable considering potential biomedical applications (in wound healing and regenerative medicine, for example) of collagen-stimulating/collagen-mimetic materials, which will be the subject of future planned investigations. 
Acknowledgements. This work was supported by EPSRC grants EP/F048114/1 and EP/G026203/1 to IWH. Use of the Chemical Analysis Facility at the University of Reading is acknowledged. We thank Professor Alison Rodger for her advice and assistance with circular and linear dichroism experiments. We also thank Dr Juan F. Miravet for his useful comments regarding the NMR spectra. 


\section{References}

1. Accardo. A, Tesauro. D, Mangiapia. D, Pedone. C \& Morelli, Peptide Science, 2006, $88,115-121$.

2. Kokkoli. E, Mardilovich. A, Wedekind. A, Rexeisen. E.L, Garg.A \& Craig. J.A, Soft Matter, 2006, 2, 1015 - 1024.

3. Paramonov. S.E, Jun. H.W \& Hartgerink. J.D, JACS, 2006, 128, $7291-7298$.

4. Cui. H, Webber. M.J \& Stupp. S.I, Peptide Science, 2009, 94, 1- 18.

5. Hamley. I.W, Soft Matter, 2011, 7, 4122 - 4138.

6. Dennis. W.P, Lowik. M \& van Hest. J.C.M, Chem. Soc. Rev., 2004, 33, $234-245$.

7. Zhao. X, Pan. F, Xu. H, Yassen. M, Shan. H, Hausser C.A.E, Zhang. S \& Lu, Chem Soc Rev., 2010, 39, 3480 - 3498.

8. Adams. D.J, Holtzmann. K, Schnieder. C \& Butler. M.F, Langmuir, 2007, 23, 1272912736.

9. Verch. A, Hahn. H, Krause. E, Colfenac. H \& Borner. H.G, ChemComm., 2010, 46, 8938-8940.

10. Cui. H, Muraoka. T, Cheetham. A \& Stupp. S.I, Nano Lett., 2009, 9, 945 - 951.

11. Lowik. D.W.P.M. van Hest. J.C.M, Chem. Soc. Rev., 2004, 33, $234-245$.

12. Aulisa. L, Forraz. N, McGuckin. C \& Hartgerink. J.D, Acta Biomaterialia., 2008, 5, 842853.

13. Castelletto. V, Hamley. I.W, Perez. J, Abezgauz. L \& Danino. D, Chem. Commun., 2010, 46, $9185-9187$.

14. V. Castelletto, I. W. Hamley, J. Adamcik, R. Mezzenga and J. Gummel, Soft Matter, 2012, 8, 217-226.

15. Manet. S, Lecchi. A, Imperor-Clerc. M, Zholobenko. V, Durand. D, Oliveira. C.L.P, Pederson. J.S, Grillo. I, Menau. F and Rochas. J. Phys. Chem. B, 2011, 115, $11318-$ 11329.

16. Alexandridis. P and Lindman. B, (Eds) 'Amphiphilic Block Copolymers: Self-assembly and Applications', Elsevier, Amsterdam, 2000. 
17. Hamley. I.W. "Block Copolymers in Solution", Wiley (2005).

18. Katayama. K, Armendariz-Borunda. J, Raghow. R, Kang. A.H \& Seyer. J.M, THE JOURNAL OF BIOLOGICAL CHEMISTRY, 1993, 268, 9941-9944.

19. Alexandridis. P, Holzwarth. J.F and Hatton. A.T, Macromolecules, 1994, 27, 2414 2425.

20. G. Wanka, H. Hoffmann, and W. Ulbricht, Macromolecules, 1994, 27, 4145.

21. G.E.Newby, I.W.Hamley, S.M.King, C.M.Martin, N.J.Terrill, J. Colloid Interface Sci., 2009, 329, 54-61.

22. http://kur.web.psi.ch/sans1/SANSSoft/sasfit.html, in 2012.

23. Hamley. I. W.\& Krysmann. M. J, Langmuir, 2008, 24, $8210-8214$.

24. Krysmann, M. J, Hamley. I. W, Funari. S. S., Canetta, E, Macromol. Chem. Phys., 2008, 209, 883 - 889.

25. Jackson. M \& Mantsch. H. H, Critical Reviews in Biochemistry and Molecular Biology, 1995, 30(2), 95-120.

26. Childers, W. S.; Mehta, A. K.; Lu, K.; Lynn, D. G, J. Am. Chem. Soc. 2009, 131 (29), 10165-10172.

27. Bulheller, B. M.; Rodger, A.; Hirst, J. D, Phys. Chem. Chem. Phys. 2007, 9 (17), 20202035.

28. Rodger, A.; Marrington, R.; Geeves, M. A.; Hicks, M.; de Alwis, L.; Halsall, D. J.; Dafforn, T. R, Phys. Chem. Chem. Phys. 2006, 8 (27),3161-3171.

29. Kriz. J \& Dybal. J, J. Phys. Chem., 2010, 114, 3140 - 3151.

30. M. Takeda, T. Kusano, T. Matsunaga, H. Endo, M. Shibayama and T. Shikata, Langmuir, 2011, 27, 1731-1738.

31. C. A. Dreiss, Soft Matter, 2007, 3, 956-970. 
Table 1. Spacings from X-ray Diffraction Experiments

\begin{tabular}{|c|c|c|c|c|c|c|}
\hline $\begin{array}{ll} & \text { Sample } \\
\text { Peak } & \end{array}$ & $\begin{array}{l}0 \% \\
\text { P123 }\end{array}$ & $\begin{array}{l}5 \% \\
\text { P123 }\end{array}$ & $\begin{array}{l}\mathbf{1 0 \%} \\
\mathrm{P} 123\end{array}$ & $\begin{array}{l}15 \% \\
\text { P123 }\end{array}$ & $\begin{array}{l}20 \% \\
\text { P123 }\end{array}$ & $\begin{array}{l}25 \% \\
\text { P123 }\end{array}$ \\
\hline $\begin{array}{l}\text { Bilayer spacing } \\
2^{\text {nd }} \text { order }+/-0.2\end{array}$ & 26.4 & 26.1 & 26.2 & & & \\
\hline 16-17 A spacing & & $\begin{array}{c}16.8 \\
\left(3^{\text {rd }}\right. \\
\text { order }) \\
\end{array}$ & $\begin{array}{c}17.4 \\
\left(3^{\text {rd }}\right. \\
\text { order }) \\
\end{array}$ & & & \\
\hline $\begin{array}{l}13-14 \AA \\
\text { spacing }\end{array}$ & 13.92 & & & & & \\
\hline $11 \AA$ spacing & 11.3 & & 10.8 & & & \\
\hline 7-9 А spacing & $\begin{array}{l}9.1 / 7.7 / \\
6.7 / 6.5\end{array}$ & $\begin{array}{c}9.8 / 9.1 \\
/ 8.4\end{array}$ & $\begin{array}{c}9.6 / 9.2 \\
/ 8.4\end{array}$ & 9.1 & & 9.1 \\
\hline $6 \AA$ spacing & & & 6.7 & $6.6 / 6.0$ & & 6.0 \\
\hline$\beta$-strand spacing & 4.78 & 4.78 & 4.75 & 4.75 & 4.75 & 4.72 \\
\hline
\end{tabular}




\section{Figures}

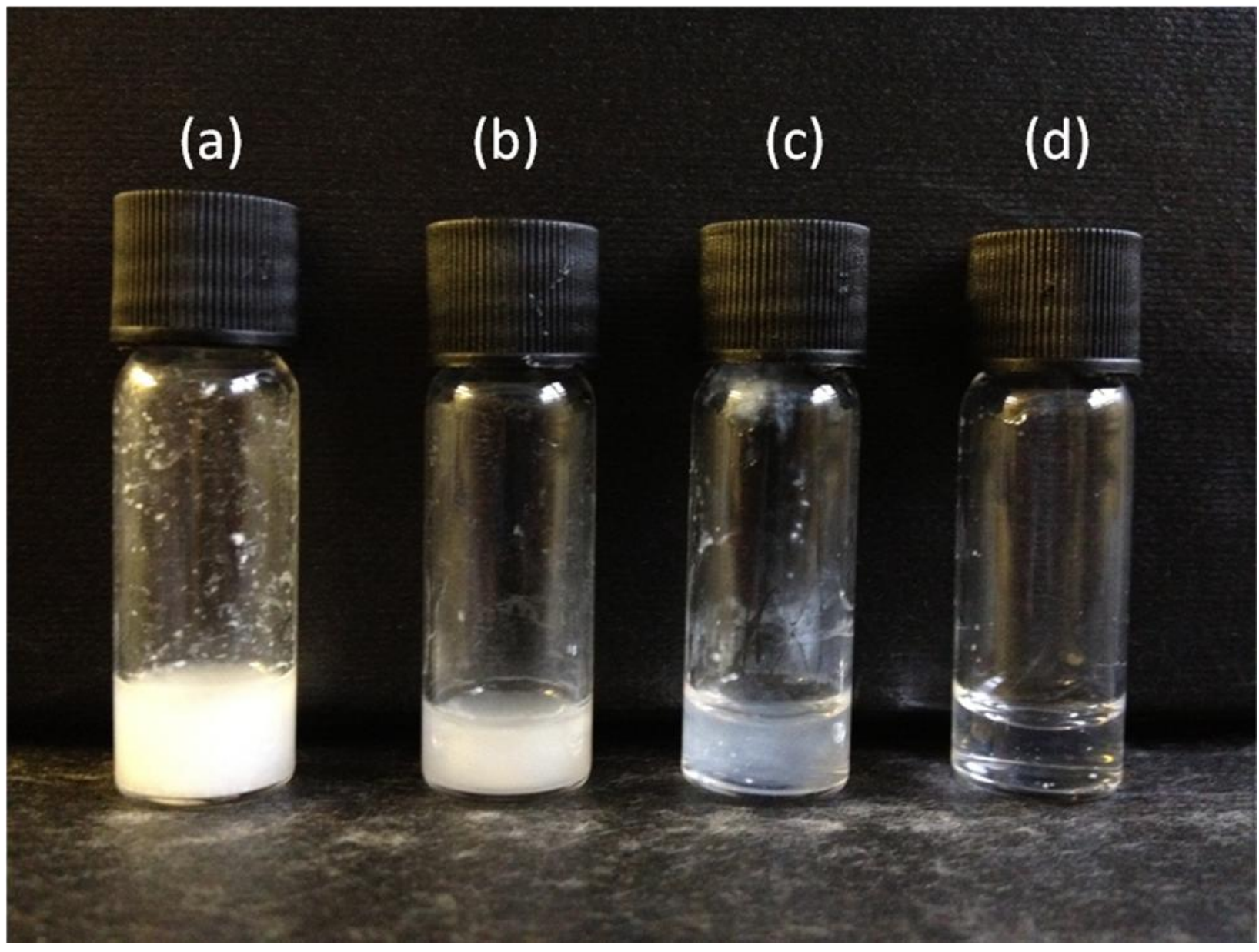

Fig. 1. Vials containing solutions of $1 \mathrm{wt} \% \mathrm{C}_{16}$-KTTKS mixed with (a) $0.2 \mathrm{wt} \%$, (b) $5 \mathrm{wt} \%$, (c) $10 \mathrm{wt} \%$, (d) $20 \mathrm{wt} \%$ p123. 

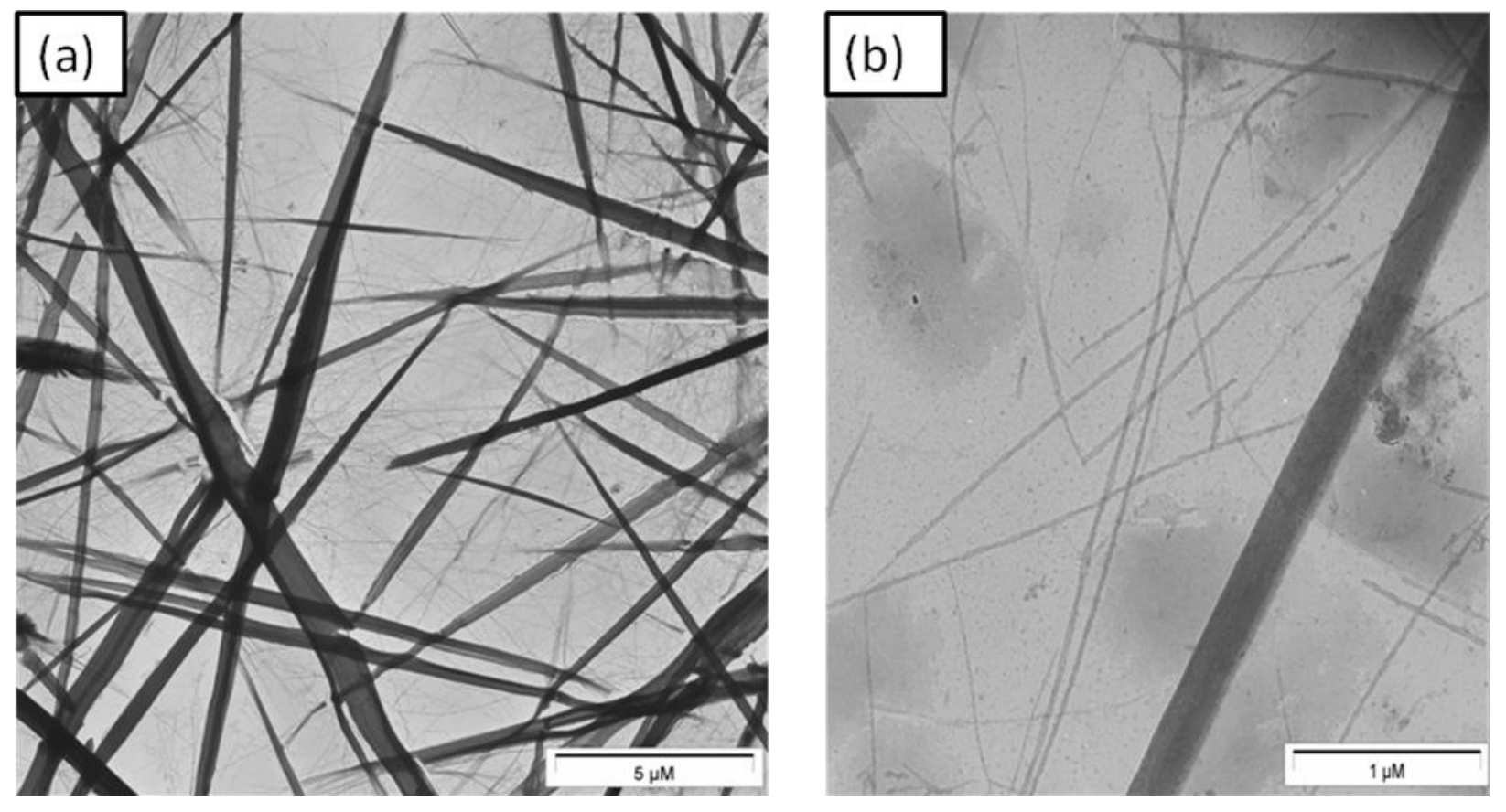

Fig. 2. TEM images of solutions containing $1 \mathrm{wt} \% \mathrm{C}_{16}$-KTTKS with added P123 (a) 0.2 wt $\%$, (b) $20 \mathrm{wt} \%$.



Fig.3. SANS profile of $1 \mathrm{wt} \% \mathrm{C}_{16}$-KTTKS and $20 \mathrm{wt} \%$ P123 under shear at $5 \mathrm{rpm}$ and at rest, with a fit to a cylinder form factor as described in the text. 


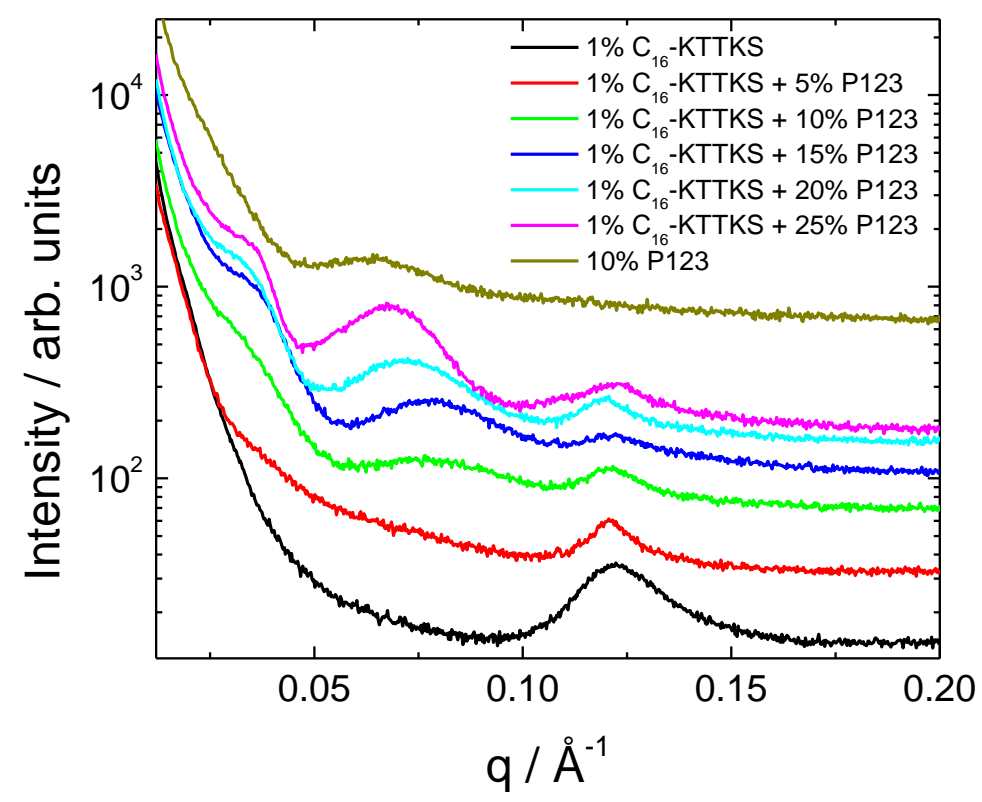

Fig. 4. SAXS profiles of $1 \mathrm{wt} \% \mathrm{C}_{16}$-KTTKS with varying P123 concentrations (indicated). 


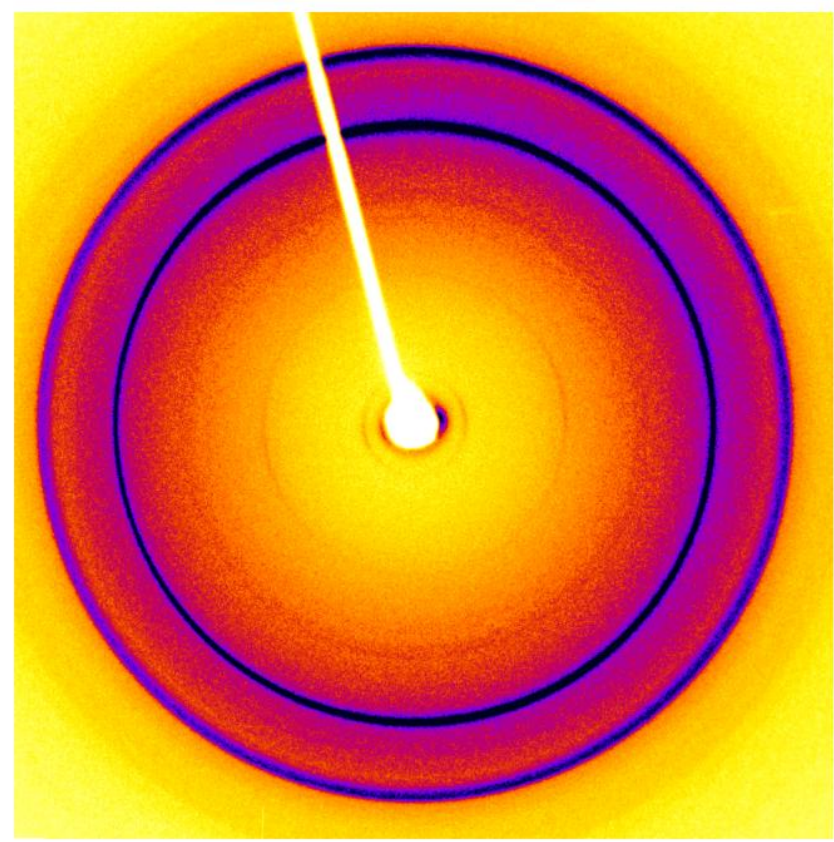

Fig. 5. Fibre x-ray diffraction pattern of $1 \mathrm{wt} \% \mathrm{C}_{16}-\mathrm{KTTKS}+15 \mathrm{wt} \% \mathrm{P} 123$. 


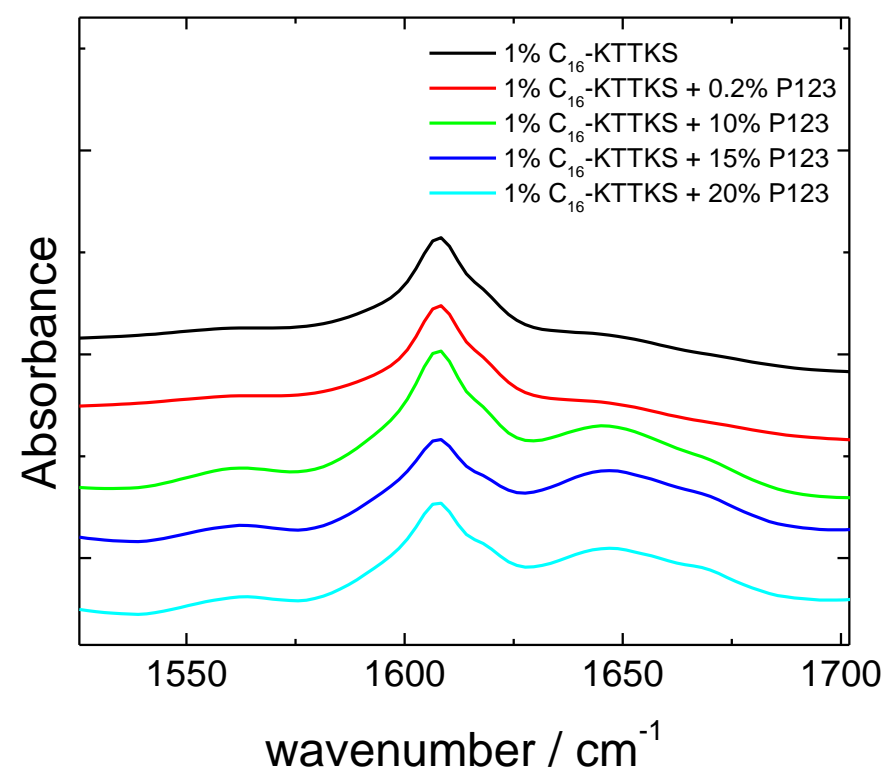

Fig. 6. FTIR spectra in the amide I' band for solutions containing mixtures of $1 \mathrm{wt} \%$ Matrixyl and P123. 

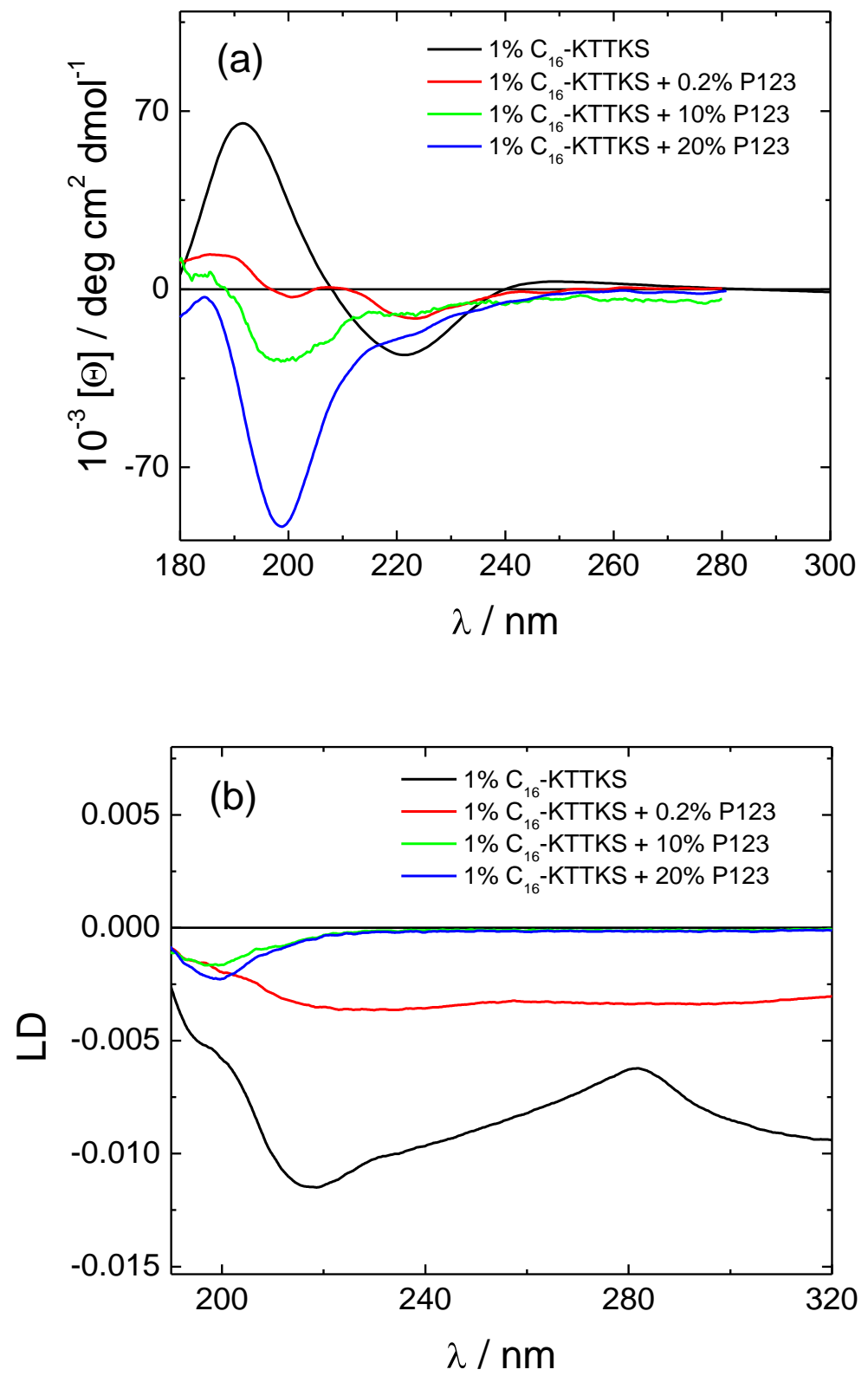

Fig. 7. (a) Circular dichroism spectra from a $1 \mathrm{wt} \%$ solution of $\mathrm{C}_{16^{-}} \mathrm{KTTKS}$ and $1 \mathrm{wt} \% \mathrm{C}_{16^{-}}$ KTTKS mixtures with varying concentrations of P123 as indicated. (b) Linear dichroism spectra for the same mixtures. 



Fig. 8. SAXS profiles for order of mixing and ageing experiments for (a) $1 \mathrm{wt} \% \mathrm{C}_{16}-\mathrm{KTTKS}$ $+5 \mathrm{wt} \% \mathrm{P} 123$, (b) $1 \mathrm{wt} \% \mathrm{C}_{16}$-KTTKS + $20 \mathrm{wt} \% \mathrm{P} 123$ and (c) form factor fitting for $1 \mathrm{wt} \%$ $\mathrm{C}_{16}$-KTTKS + $5 \mathrm{wt} \% \mathrm{P} 123\left(\mathrm{C}_{16}\right.$-KTTKS added later) and $1 \mathrm{wt} \% \mathrm{C}_{16}$-KTTKS + $20 \mathrm{wt} \% \mathrm{P} 123$ (P123 added later - aged). (d) SAXS profiles from thermal treatment (heating to $60{ }^{\circ} \mathrm{C}$, then cooling to room temperature) for $1 \mathrm{wt} \% \mathrm{C}_{16}$-KTTKS $+20 \mathrm{wt} \% \mathrm{P} 123$. 